\title{
A Non-orthogonal Joint Coordinate System for the Calculation of Anatomically Practical Joint Torque Power in Three-dimensional Hip Joint Motion
}

\author{
Natsuki Sado',2*, Shinsuke Yoshioka ${ }^{1}$ and Senshi Fukashiro ${ }^{1}$ \\ ${ }^{1}$ Department of Life Sciences, The University of Tokyo, Tokyo, Japan. \\ 3-8-1, Komaba, Meguro-ku, Tokyo, 153-8902 Japan \\ E-mail: sado@g.ecc.u-tokyo.ac.jp \\ ${ }^{2}$ Research Fellow of the Japan Society for the Promotion of Science, Tokyo, Japan \\ Kojimachi Business Center, 5-3-1, Kojimachi, Chiyoda-ku, Tokyo, 153-8902 Japan \\ [Received April 16, 2017; Accepted September 21, 2017; Published online October 11, 2017]
}

\begin{abstract}
We examine the advantages of a non-orthogonal joint coordinate system (JCS) in calculating each anatomical torque's power through comparison with a segment coordinate system (SCS) of the distal segment. To clarify the differences between coordinate systems, kinematic data were collected from 12 male participants swinging their legs laterally and anteriorly under two conditions: the toe facing forward and facing laterally. The mechanical power and work exerted by each hip anatomical torque in JCS and SCS were calculated. With the toe facing forward, there is no significant difference between the two methods. The largest energy generators were abduction torque for lateral swing and flexion torque for anterior swing. With the toe facing laterally, in JCS, these results were consistent for both lateral swing (abduction: $0.21 \pm 0.06$ $\mathrm{J} / \mathrm{kg}$; flexion: $0.06 \pm 0.04 \mathrm{~J} / \mathrm{kg}$ ) and anterior swing (flexion: $0.35 \pm 0.09 \mathrm{~J} / \mathrm{kg}$; adduction: $0.01 \pm$ $0.01 \mathrm{~J} / \mathrm{kg}$ ). However, in SCS, the largest energy generator for lateral swing changed from abduction $(0.08 \pm 0.07 \mathrm{~J} / \mathrm{kg})$ to flexion torque $(0.22 \pm 0.12 \mathrm{~J} / \mathrm{kg})$. For anterior swing, the hip adduction torque generated as large energy $(0.14 \pm 0.08 \mathrm{~J} / \mathrm{kg})$ as hip flexion torque $(0.20 \pm 0.08$ $\mathbf{J} / \mathbf{k g}$ ) in SCS. Therefore, although SCS resulted in an inconsistency between power generator and movement due to hip external rotation, JCS avoided it, regardless of leg position, allowing JCS to investigate the power generation/absorption of each anatomical torque, particularly during long axial rotation.
\end{abstract}

Keywords: inverse dynamics, three-dimensional analysis, expression of joint torque, mechanical work

\section{Introduction}

In biomechanics, the joint power/work calculation provides information on the function of each muscle group such as mechanical energy generator/absorber (Gordon et al., 1980). Joint torque powers well discriminated the pathological gait (Winter, 2009) and kinetical gender differences which could not be discriminated with joint torques (Ferber et al., 2003). Additionally, the contribution of each joint torque to human performances has been revealed with joint power/work variables (Bezodis et al., 2008; Fukashiro and Komi, 1987; Inaba et al., 2013). As shown in them, the mechanical power/work calculation provides useful insights regarding the biomechanical basis of human movements.
In three-dimensional analysis, researchers need to choose a coordinate system to express the joint kinetics, and joint torques have been typically transformed into the global (or laboratory) coordinate system (GCS), the segment coordinate system (SCS) of the distal segment or the non-orthogonal joint coordinate system (JCS) (Kristianslund et al., 2014). The choice of coordinate system causes significant differences in the calculation results (Kristianslund et al., 2014; Schache et al., 2008; Schache and Baker, 2007). For example, Kristianslund et al. (2014) observed that the selection of the coordinate system changes the ranking of participants based on the maximum knee and hip joint torques during high-impact movements. Schache et al. (2008) reported that the effect of gait modification, such as the toe-out and medial-thrust gaits, on the calcu- 
lated value of the knee abduction torque depends on the coordinate system. These findings indicate that depending on the coordinate system, the anatomical interpretations are changeable in investigating mechanically the same phenomena, although all methods do not have any mathematical error. Therefore, it is necessary for the researchers to consider the effect of choosing the coordinate system on results.

Such options would occur while investigating the mechanical power of each anatomical torque, and JCS may be one of the important options. The JCS approach indicates the rotational relation between segments in three angles corresponding to anatomical terms, which improves the interpretation of kinematic data for both researchers and clinicians (Grood and Suntay, 1983; Wu et al., 2002). Because of these advantages, JCS has been mainly used to describe a joint angle (Desroches et al., 2010; Kristianslund et al., 2014; Wu et al., 2002). The transformation of joint torque in JCS enables the axes of joint torque and angle to be consistent (e.g. to ensure that a flexion torque will result in joint rotation around flexion axis), as suggested in previous studies (Desroches et al., 2010; Kristianslund et al., 2014; Schache and Baker, 2007). Therefore, while calculating anatomical power, utilization of JCS may prevent contradictions such as hip flexion torque generates large power/energy without hip flexion displacement, leading the analysis with consistencies between kinematics, kinetics and energetics.

However, JCS has not yet been used to investigate each anatomical torque power. This study aims at determining the advantages of JCS in calculating the power of each anatomical torque through a comparison with SCS.

\section{Methods}

\subsection{Calculation of mechanical power by each ana- tomical torque}

The hip JCS is defined as follows. The $\boldsymbol{e}_{h i p, F E}$ (flexion-extension) axis is the medial-lateral axis of the pelvic segment, the $\boldsymbol{e}_{\text {hip,IER }}$ (internal-external rotation) axis is the long axis of the thigh segment and the $\boldsymbol{e}_{h i p, A A}$ (abduction-adduction) axis is the cross product of the $\boldsymbol{e}_{\text {hip,IER }}$ and $\boldsymbol{e}_{\text {hip,FE }}$ axes (Fig. 1a).

In this study, the joint torques expressed in the
JCS were defined as the three torques along the JCS axes that add up to the total hip joint torque vector $\boldsymbol{n}_{\text {hip }}$ (Desroches et al., 2010):

$$
\begin{aligned}
\boldsymbol{n}_{\text {hip }}= & \tau_{\text {hip,FE }} \boldsymbol{e}_{\text {hip,FE }}+\tau_{\text {hip,AA }} \boldsymbol{e}_{\text {hip,AA }} \\
& +\tau_{\text {hip,IER }} \boldsymbol{e}_{\text {hip,IER }}
\end{aligned}
$$

where $\tau_{h i p, F E}, \tau_{h i p, A A}$ and $\tau_{\text {hip,IER }}$ were hip flexionextension, abduction-adduction and internal-external rotation torque components in JCS. The matrix form of Eq. (1) was

$$
\boldsymbol{n}_{h i p}=\left[\begin{array}{ll}
\boldsymbol{e}_{h i p, F E} & \boldsymbol{e}_{h i p, A A} \boldsymbol{e}_{h i p, I E R}
\end{array}\right]\left[\begin{array}{c}
\tau_{h i p, F E} \\
\tau_{h i p, A A} \\
\tau_{h i p, I E R}
\end{array}\right]
$$

From Eq. (1)', each joint torque component in JCS was calculated as shown below:

$$
\left[\begin{array}{l}
\tau_{h i p, F E} \\
\tau_{h i p, A A} \\
\tau_{h i p, I E R}
\end{array}\right]=\left[\begin{array}{lll}
\boldsymbol{e}_{h i p, F E} & \boldsymbol{e}_{h i p, A A} & \boldsymbol{e}_{h i p, I E R}
\end{array}\right]^{-1} \boldsymbol{n}_{h i p}
$$

Note that the inverse matrix and transposed matrix were not consistent because JCS axes were not orthogonal. Additionally, the hip joint torque vector $\boldsymbol{n}_{h i p}$ in thigh coordinate was as follows:

$$
\begin{aligned}
\boldsymbol{n}_{\text {hip }}= & \tau_{\text {thigh_x }} \boldsymbol{i}_{\text {thigh }}+\tau_{\text {thigh_y }} \boldsymbol{j}_{\text {thigh }} \\
& +\tau_{\text {thigh_z }} \boldsymbol{k}_{\text {thigh }}
\end{aligned}
$$

The hip joint torque power $j t p_{\text {hip }}$ can be calculated as the dot product of the joint torque vectors and the difference between the angular velocity vectors of the pelvis and thigh $\left(\omega_{\text {thigh }}-\omega_{\text {pelvis }}\right)$ :

$$
j t p_{\text {hip }}=n_{\text {hip }} \cdot \omega_{\text {hip }} \text {, where } \omega_{\text {hip }}=\omega_{\text {thigh }}-\omega_{\text {pelvis }}
$$

From Eq. (1), Eq. (4) can be deformed as follows:

$$
\begin{aligned}
j t p_{\text {hip }}= & \left(\tau_{\text {hip,FE }} \boldsymbol{e}_{\text {hip,FE }}+\tau_{\text {hip,AA}} \boldsymbol{e}_{\text {hip,AA}}+\tau_{\text {hip,IER }}\right. \\
& \left.\boldsymbol{e}_{\text {hip,IER }}\right) \cdot \omega_{\text {hip }} \\
= & \left(\tau_{\text {hip,FE }} \boldsymbol{e}_{\text {hip,FE }}\right) \cdot \omega_{\text {hip }}+\left(\tau_{\text {hip,AA }} \boldsymbol{e}_{\text {hip,AA }}\right) \\
& \cdot \omega_{\text {hip }}+\left(\tau_{\text {hip,IER }} \boldsymbol{e}_{\text {hip,IER }}\right) \cdot \omega_{\text {hip }}
\end{aligned}
$$

Therefore, the mechanical powers exerted by each anatomical torque (extension-flexion power $\left(j t p_{\text {hip,FEJCS }}\right), \quad$ abduction-adduction power $\left(j t p_{h i p, A A_{-} J C S}\right)$ and internal-external rotation power $\left.\left(j t p_{\text {hip,IER_JCS }}\right)\right)$ were calculated as follows:

$$
\begin{aligned}
& j t p_{h i p}=j t p_{h i p, F E_{-} J C S}+j t p_{h i p, A A_{-} J C S}+j t p_{\text {hip,IER_JCS }} \\
& j t p_{\text {hip,FE_JCS }}=\left(\tau_{\text {hip,FE }} \boldsymbol{e}_{\text {hip,FE }}\right) \cdot \omega_{\text {hip }} \\
& j t p_{\text {hip,AA_JCS }}=\left(\tau_{\text {hip,AA }} \boldsymbol{e}_{\text {hip, AA }}\right) \cdot \omega_{\text {hip }} \\
& j t p_{\text {hip,IER_JCS }}=\left(\tau_{\text {hip,IER }} \boldsymbol{e}_{\text {hip,IER }}\right) \cdot \omega_{\text {hip }}
\end{aligned}
$$

The hip joint torque power calculation in thigh coordinate is as follows:

$$
\begin{aligned}
j t p_{\text {hip }}= & \boldsymbol{n}_{\text {hip }} \cdot \omega_{\text {hip }} \\
= & \tau_{\text {thigh_x }} \omega_{\text {hip_thigh_x }}+\tau_{\text {thigh_y }} \omega_{\text {hip_thigh_y }} \\
& +\tau_{\text {thigh_z }} \omega_{\text {hip_thigh_z }}
\end{aligned}
$$

Therefore, each anatomical torque power in SCS 
a. Definition of the hip joint coordinate system

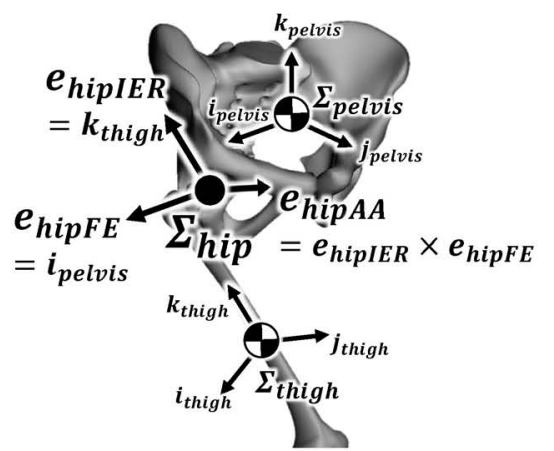

b. Definitions of the segment coordinate systems

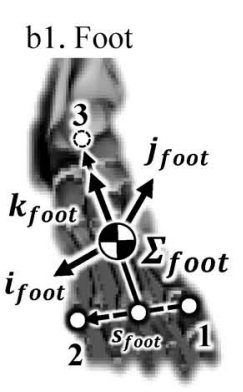

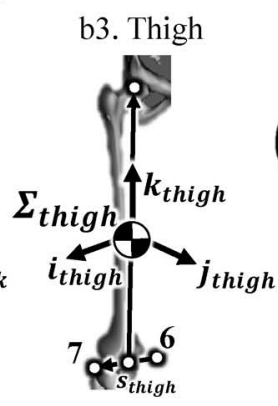

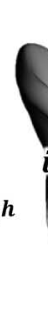

b4. Pelvis

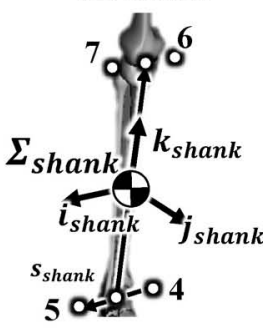

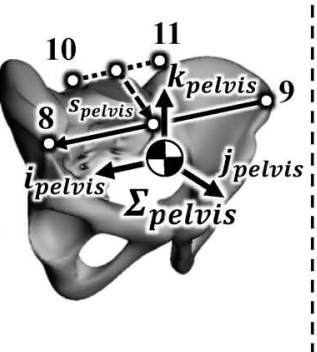

c. Placements of Markers

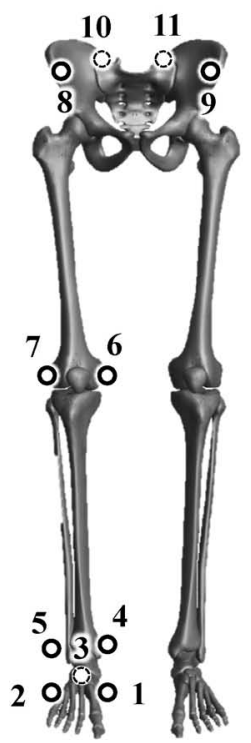

Fig. 1 The method used to define the (a) hip joint and (b) segment coordinate systems (JCS and SCSs), and (c) placements of markers used in experiment.

1 (2): 1st (5th) Metatarsal Heads, 3: Calcaneous, 4 (5):Medial (Lateral) Malleolus, 6 (7): Medial (Lateral) side of knee joint, 8 (9) Right (Left) Antero-Superior Iliac Spines, 10 (11) Right (Left) Postero-Superior Iliac Spines, $\Sigma_{\text {foot }}$ : foot SCS, $\Sigma_{\text {shank }}$ : shank SCS, $\Sigma_{\text {thigh }}$ : thigh SCS, $\Sigma_{\text {pelvic }}$ : pelvic SCS, $\Sigma_{\text {hip }}$ : hip JCS.

method was as follows:

$$
\begin{aligned}
& j t p_{h i p}=j t p_{h i p, F E \_S C S}+j t p_{\text {hip,AA_SCS }}+j t p_{\text {hip,IER_SCS }} \\
& j t p_{\text {hip,FE_SCS }}=\tau_{\text {thigh_x }} \omega_{\text {hip_thigh_x }} \\
& j t p_{\text {hip,AA_SCS }}=\tau_{\text {thigh_y }} \omega_{\text {hip_thigh_y }} \\
& j t p_{\text {hip,IER_SCS }}=\tau_{\text {thigh_z }} \omega_{\text {hip_thigh_z }}
\end{aligned}
$$

Note that $\omega_{\text {hip }}$ in thigh coordinate was as follows:

$$
\begin{aligned}
\omega_{\text {hip }}= & \omega_{\text {hip_thigh_x }} \boldsymbol{i}_{\text {thigh }}+\omega_{\text {hip_thigh_y }} \boldsymbol{j}_{\text {thigh }} \\
& +\omega_{\text {hip_thigh_z }} \boldsymbol{k}_{\text {thigh }}
\end{aligned}
$$

\subsection{Experimental protocol}

Twelve male participants ( $26.5 \pm 4.1$ years; $1.76 \pm$ $0.06 \mathrm{~m} ; 69.3 \pm 9.5 \mathrm{~kg}$ ) swung their right leg laterally and anteriorly until their right feet reached approximately left knee height under two conditions: the right toe facing forward and the right toe facing laterally. Three trials of each direction under each condition were performed, and kinetic analysis was performed for all trials. Written informed consent was obtained from all participants before performing the experiments.

The kinematic and ground reaction force (GRF) data were collected using a 13-camera motion cap- ture system at a sampling rate of $200 \mathrm{~Hz}$ (Motion Analysis Corporation, Santa Rosa, CA, USA) and a force platform at a sampling rate of $2000 \mathrm{~Hz}$ (Force Plate 9281E, Kistler, Winterthur, Switzerland). Eleven markers were used for the analysis (Fig. 1c). Position coordinates of the markers were smoothed using a fourth-order two-directional Butterworth low-pass digital filter. Residual analysis (Wells and Winter, 1980; Winter, 2009) was performed to identify the cut-off frequency of the three-dimensional position of each marker in each trial, with a range of cut-offs between 10.0 and 20.0 $\mathrm{Hz}$ ultimately used for the dataset. The current study defined the analysis phase as the period from the instant of toe-off to the instants of maximum hip abduction for lateral swing and maximum flexion for anterior swing. The instant of toe-off was determined by the GRF signal.

The inertial parameters for each segment were estimated using anthropometric data from Dumas et al. (Dumas et al., 2007). A four-segment biomechanical model (pelvis, thigh, shank and foot in swing leg) was used to calculate joint kinematics 
and kinetics. The knee and ankle joint centers were localized at the midpoint of the lateral and medial markers. The hip joint center was estimated using the method recommended by Harrington et al. (Harrington et al., 2007). Methods are shown in Fig. 1b; in brief, the SCSs of the right foot, right shank, right thigh, and pelvis were defined using the coordinates of the markers and joint centers. Then, the hip JCS was defined using the SCSs of the right thigh and pelvis (Fig. 1a). The hip joint angles (hip flexion-extension angle $\left(\theta_{\text {hip,FE }}\right)$, abduction-adduction angle $\left(\theta_{\text {hip,AA}}\right)$ and internal-external rotation angle $\left(\theta_{\text {hip,IER }}\right)$ ) were calculated as follows (Grood and Suntay, 1983):

$$
\begin{aligned}
& \theta_{\text {hip,FE }}=\pi / 2-\cos ^{-1}\left(\boldsymbol{k}_{\text {pelvis }} \cdot \boldsymbol{e}_{\text {hip,AA }}\right) \\
& \theta_{\text {hip,AA }}=\pi / 2-\cos ^{-1}\left(\boldsymbol{k}_{\text {thigh }} \cdot \boldsymbol{i}_{\text {pelvis }}\right) \\
& \theta_{\text {hip,IER }}=\pi / 2-\cos ^{-1}\left(\boldsymbol{i}_{\text {thigh }} \cdot \boldsymbol{e}_{\text {hip,AA }}\right)
\end{aligned}
$$

Individual joint angular velocities were calculated as a time derivative of joint angle profiles. The inverse dynamics method (Winter, 2009) was used to calculate the three-dimensional hip joint torque. The hip joint torque was transformed in SCS and JCS. The joint torque power exerted by each torque in SCS and JCS was calculated. The positive and negative works were calculated as an integral of positive and negative power, respectively.

\subsection{Statistical analysis}

In statistical analysis, the mean of the data from three trials was used as the representative value for each participant. The data were expressed as mean \pm SD. The Shapiro-Wilk test was performed to test the normality of distribution. The paired $t$-test or Wilcoxon signed-rank test was used to determine the differences in peak torque, peak power and positive work between the JCS and SCS methods. Statistical significance was set at $p<0.01$.

\section{Results}

In lateral swing, the displacement of hip flexion $(+)$ /extension $(-)$ angle and abduction $(+) / \mathrm{ad}-$ duction $(-)$ angle were $16.8^{\circ} \pm 11.5^{\circ}$ and $33.8^{\circ} \pm$ $5.4^{\circ}$ with the toe facing forward, and $20.0^{\circ} \pm 11.9^{\circ}$ and $34.5^{\circ} \pm 6.2^{\circ}$ with the toe facing laterally, respectively. In terms of anterior swing, they were $54.1^{\circ} \pm 7.3^{\circ}$ and $1.8^{\circ} \pm 6.4^{\circ}$ with the toe facing forward, and $53.1^{\circ} \pm 9.5^{\circ}$ and $-4.2^{\circ} \pm 7.4^{\circ}$ with the toe facing laterally, respectively.

There were significant differences in the peak powers and positive works between the two coordinate systems with the toe facing laterally, while no

\begin{tabular}{|c|c|c|c|c|c|c|}
\hline \multirow{2}{*}{ Variables } & \multicolumn{3}{|c|}{ Toe facing forward } & \multicolumn{3}{|c|}{ Toe facing lateral } \\
\hline & JCS & SCS & & JCS & SCS & \\
\hline \multicolumn{7}{|l|}{ Lateral-Swing } \\
\hline \multicolumn{7}{|l|}{ Flexion } \\
\hline$T_{\text {peak }}$ & $0.13 \pm 0.11 \mathrm{Nm} / \mathrm{kg}$ & $0.19 \pm 0.13 \mathrm{Nm} / \mathrm{kg}$ & n.s. & $0.26 \pm 0.14 \mathrm{Nm} / \mathrm{kg}$ & $0.50 \pm 0.12 \mathrm{Nm} / \mathrm{kg}$ & * \\
\hline$P_{\text {peak }}$ & $0.14 \pm 0.13 \mathrm{~W} / \mathrm{kg}$ & $0.22 \pm 0.22 \mathrm{~W} / \mathrm{kg}$ & n.s. & $0.24 \pm 0.17 \mathrm{~W} / \mathrm{kg}$ & $0.70 \pm 0.30 \mathrm{~W} / \mathrm{kg}$ & * \\
\hline$W_{\text {positive }}$ & $0.02 \pm 0.03 \mathrm{~J} / \mathrm{kg}$ & $0.05 \pm 0.07 \mathrm{~J} / \mathrm{kg}$ & n.s. & $0.06 \pm 0.05 \mathrm{~J} / \mathrm{kg}$ & $0.21 \pm 0.08 \mathrm{~J} / \mathrm{kg}$ & * \\
\hline \multicolumn{7}{|l|}{ Abduction } \\
\hline$T_{p e a k}$ & $0.61 \pm 0.20 \mathrm{Nm} / \mathrm{kg}$ & $0.60 \pm 0.20 \mathrm{Nm} / \mathrm{kg}$ & n.s. & $0.55 \pm 0.12 \mathrm{Nm} / \mathrm{kg}$ & $0.34 \pm 0.14 \mathrm{Nm} / \mathrm{kg}$ & * \\
\hline$P_{\text {peak }}$ & $0.78 \pm 0.45 \mathrm{~W} / \mathrm{kg}$ & $0.69 \pm 0.46 \mathrm{~W} / \mathrm{kg}$ & n.s. & $0.72 \pm 0.31 \mathrm{~W} / \mathrm{kg}$ & $0.24 \pm 0.18 \mathrm{~W} / \mathrm{kg}$ & * \\
\hline$W_{\text {positive }}$ & $0.21 \pm 0.05 \mathrm{~J} / \mathrm{kg}$ & $0.18 \pm 0.05 \mathrm{~J} / \mathrm{kg}$ & n.s. & $0.21 \pm 0.06 \mathrm{~J} / \mathrm{kg}$ & $0.06 \pm 0.04 \mathrm{~J} / \mathrm{kg}$ & * \\
\hline \multicolumn{7}{|c|}{ Anterior-Swing } \\
\hline$T_{\text {peak }}$ & $0.68 \pm 0.36 \mathrm{Nm} / \mathrm{kg}$ & $0.68 \pm 0.35 \mathrm{Nm} / \mathrm{kg}$ & n.s. & $0.67 \pm 0.29 \mathrm{Nm} / \mathrm{kg}$ & $0.56 \pm 0.26 \mathrm{Nm} / \mathrm{kg}$ & * \\
\hline$P_{\text {peak }}$ & $1.46 \pm 1.04 \mathrm{~W} / \mathrm{kg}$ & $1.48 \pm 1.08 \mathrm{~W} / \mathrm{kg}$ & n.s. & $1.45 \pm 0.80 \mathrm{~W} / \mathrm{kg}$ & $0.89 \pm 0.62 \mathrm{~W} / \mathrm{kg}$ & * \\
\hline$W_{\text {positive }}$ & $0.34 \pm 0.08 \mathrm{~J} / \mathrm{kg}$ & $0.34 \pm 0.08 \mathrm{~J} / \mathrm{kg}$ & n.s. & $0.35 \pm 0.09 \mathrm{~J} / \mathrm{kg}$ & $0.20 \pm 0.08 \mathrm{~J} / \mathrm{kg}$ & * \\
\hline \multicolumn{7}{|l|}{ Adduction } \\
\hline$T_{\text {peak }}$ & $0.04 \pm 0.08 \mathrm{Nm} / \mathrm{kg}$ & $0.08 \pm 0.09 \mathrm{Nm} / \mathrm{kg}$ & n.s. & $0.18 \pm 0.12 \mathrm{Nm} / \mathrm{kg}$ & $0.47 \pm 0.26 \mathrm{Nm} / \mathrm{kg}$ & * \\
\hline$P_{\text {peak }}$ & $0.07 \pm 0.01 \mathrm{~W} / \mathrm{kg}$ & $0.07 \pm 0.06 \mathrm{~W} / \mathrm{kg}$ & n.s. & $0.10 \pm 0.09 \mathrm{~W} / \mathrm{kg}$ & $0.72 \pm 0.62 \mathrm{~W} / \mathrm{kg}$ & * \\
\hline$W_{\text {positive }}$ & $0.01 \pm 0.01 \mathrm{~J} / \mathrm{kg}$ & $0.01 \pm 0.01 \mathrm{~J} / \mathrm{kg}$ & n.s. & $0.01 \pm 0.01 \mathrm{~J} / \mathrm{kg}$ & $0.14 \pm 0.08 \mathrm{~J} / \mathrm{kg}$ & * \\
\hline
\end{tabular}
significant difference was found between the

Table 1 Mean \pm SD magnitudes for the torque, power and work variables.

Note *: the significant difference between JCS and SCS, $p<0.01$

JCS: joint coordinate system/SCS: segment coordinate system/T: torque/P: power/W: work 
methods with the toe facing forward (Table 1).

In both methods, for lateral swing with the toe facing forward, the hip abduction torque exerted the largest positive power and work (Fig. 2 and Table 1). These results were consistent in the JCS method, with right toe facing laterally (Fig. 2 and Table 1). However, in the SCS method, the hip flexion torque became the largest energy generator for the lateral swing with the toe facing laterally (Fig. 2 and Table 1).

For anterior swing with the toe facing forward, in both methods, the hip flexion torque exerted positive power/work, while the power/work exerted by hip adduction torque was nearly zero (Fig. 3 and Table 1). With the toe facing laterally, although these results were consistent in JCS method, the hip adduction torque exerted as more positive power/work as the hip flexion torque in SCS method (Fig. 3 and Table 1).

Mathematically, the sum of the power by joint torques in SCS and JCS ought to be consistent with the product of the total joint torque and the difference between the angular velocities of the pelvis and thigh. The consistency of these values was confirmed (Fig. 4).

\section{Discussion}

This study aims at determining the features of JCS in calculating the power of each anatomical torque through a comparison with the SCS. The JCS and SCS methods were applied on swinging right legs laterally and anteriorly, with the toe facing forward and facing laterally. We confirmed that the difference between the joint torque power in JCS and SCS was actually large when the long axial rotation occurred.

The participants performed the same movement by swinging their right leg laterally and anteriorly with and without hip external rotation. Using JCS,
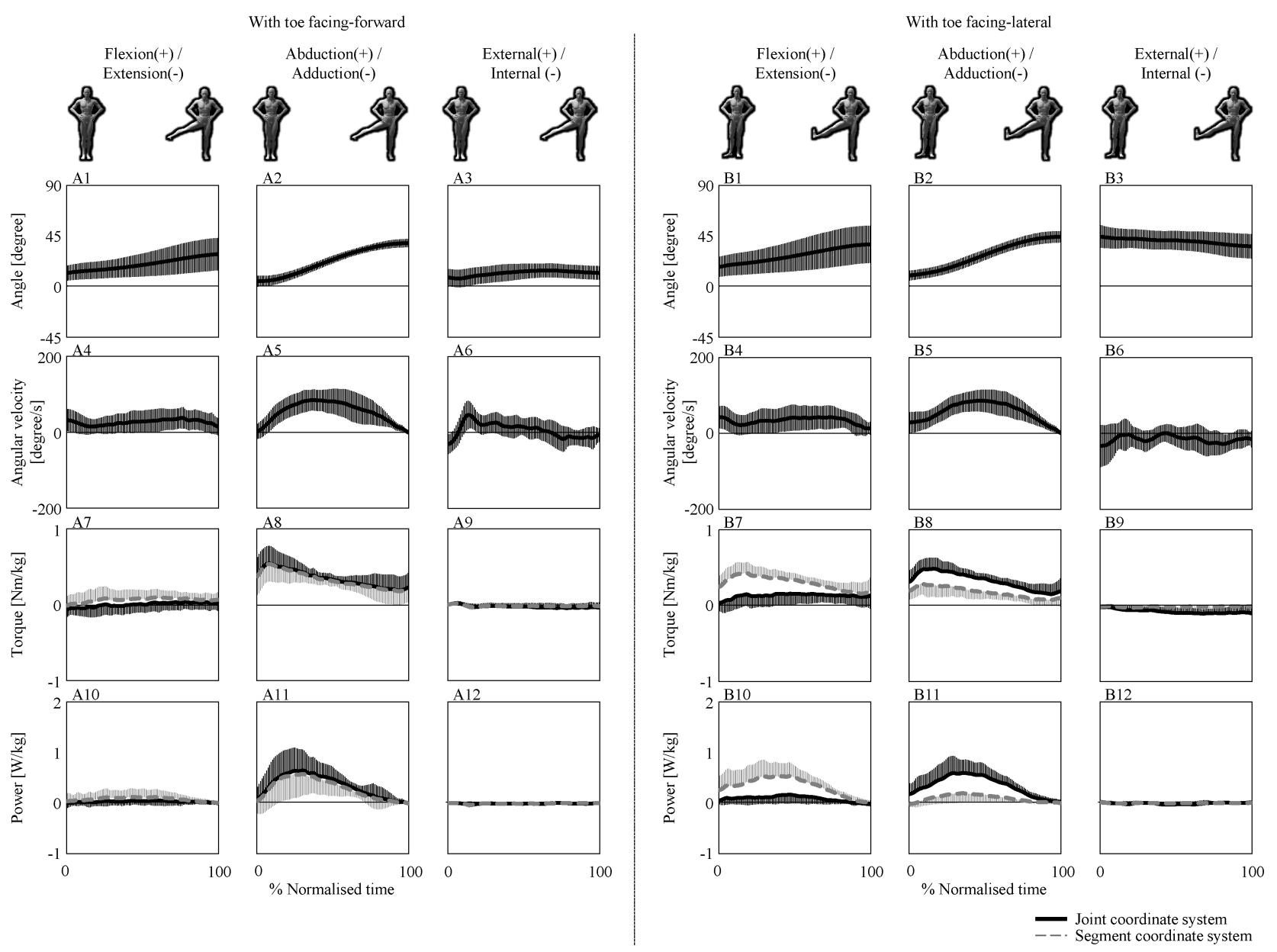

Fig. 2 Ensemble averages of the hip joint angle, angular velocity, torque and torque power during lateral swing of the leg with toe facing forward and facing laterally. 

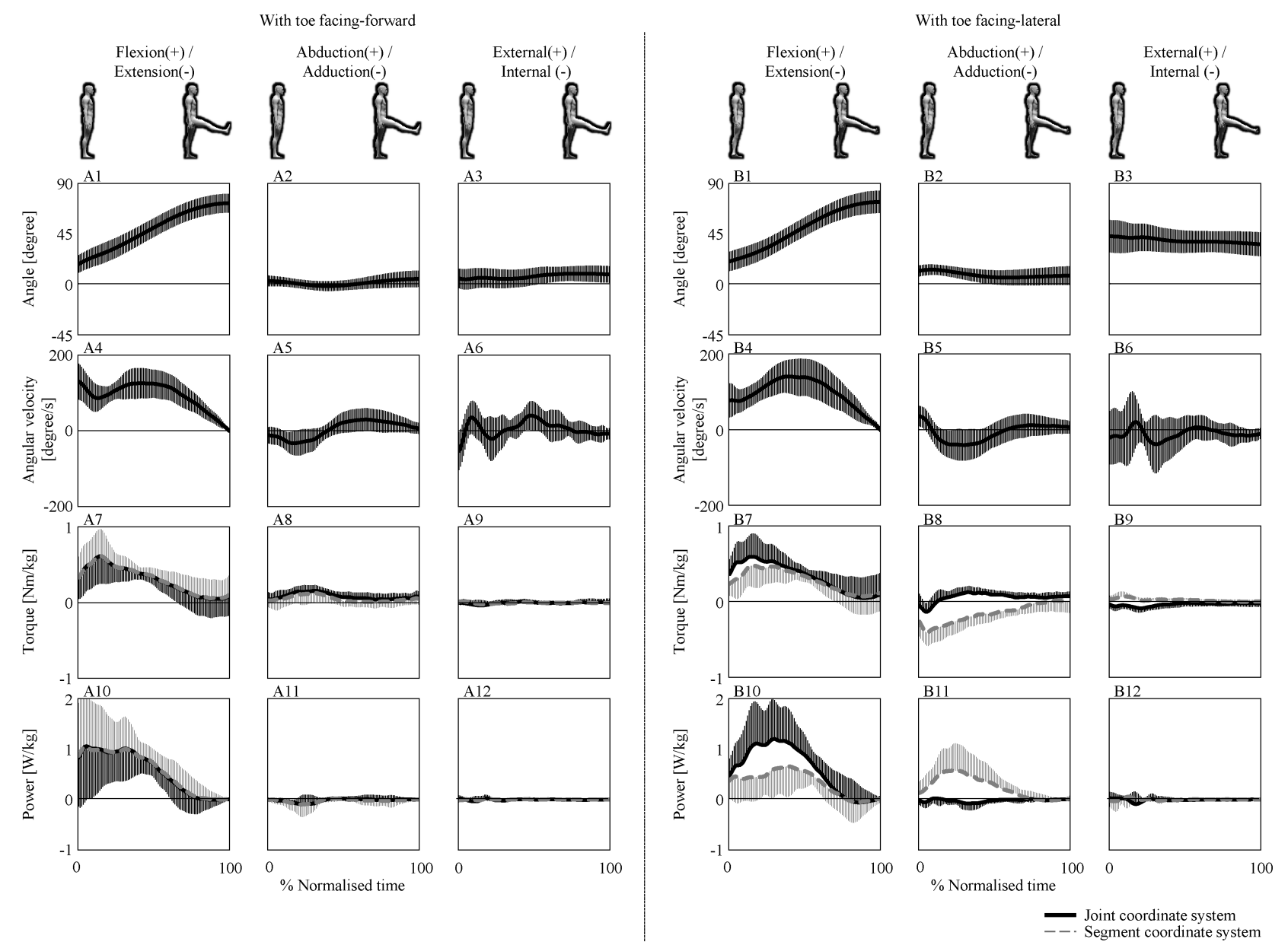

Fig. 3 Ensemble averages of the hip joint angle, angular velocity, torque and torque power during anterior swing of the leg with toe facing forward and facing laterally.

it was observed that hip abduction and flexion torque contributed maximally to energy generation in both leg positions for lateral swing and anterior swing, respectively (Figs. 2, 3 and Table 1). Using the SCS with the toe facing forward, these results are consistent for both swings (Figs. 2, 3 and Table 1). However, using the SCS for lateral swing with the toe facing laterally, the largest energy generator changed from hip abduction to flexion torque (Fig. 2 and Table 1). For anterior swing with the toe facing laterally, the hip adduction torque exerted as large power/work as hip flexion torque (Fig. 3 and Table 1). The change in the contributor was caused by hip external rotation. With increase in hip external rotation, the SCS medial-lateral axis direction approached the JCS anterior-posterior axis direction, as shown in Fig. 5, which resulted in an increase in the mechanical power/work exerted by the hip flexion torque for lateral swing and by the hip adduction torque for anterior swing in the SCS methods. This result indicates that the choice of the coordinate system critically affects the conclusion on the contribution of each joint torque to energy generation.

Note that the powers exerted by each joint torque on both SCS and JCS were 'mathematically' correct; the differences between them did not represent errors. However, the largest contributor to power generation changed with the change in the coordinate system (Figs. 2, 3 and Table 1). The experimental movement in this study was swinging the leg laterally and anteriorly, and the kinematic results indicated that the anatomical motions with the largest angular displacements were hip abduction for lateral swing and hip flexion for anterior swing, regardless of the hip external rotation (Figs. 2, 3). Therefore, the largest contributor should be hip abduction torque for lateral swing and flexion torque for anterior swing, regardless of the hip external rotation, as described by the JCS method. If 


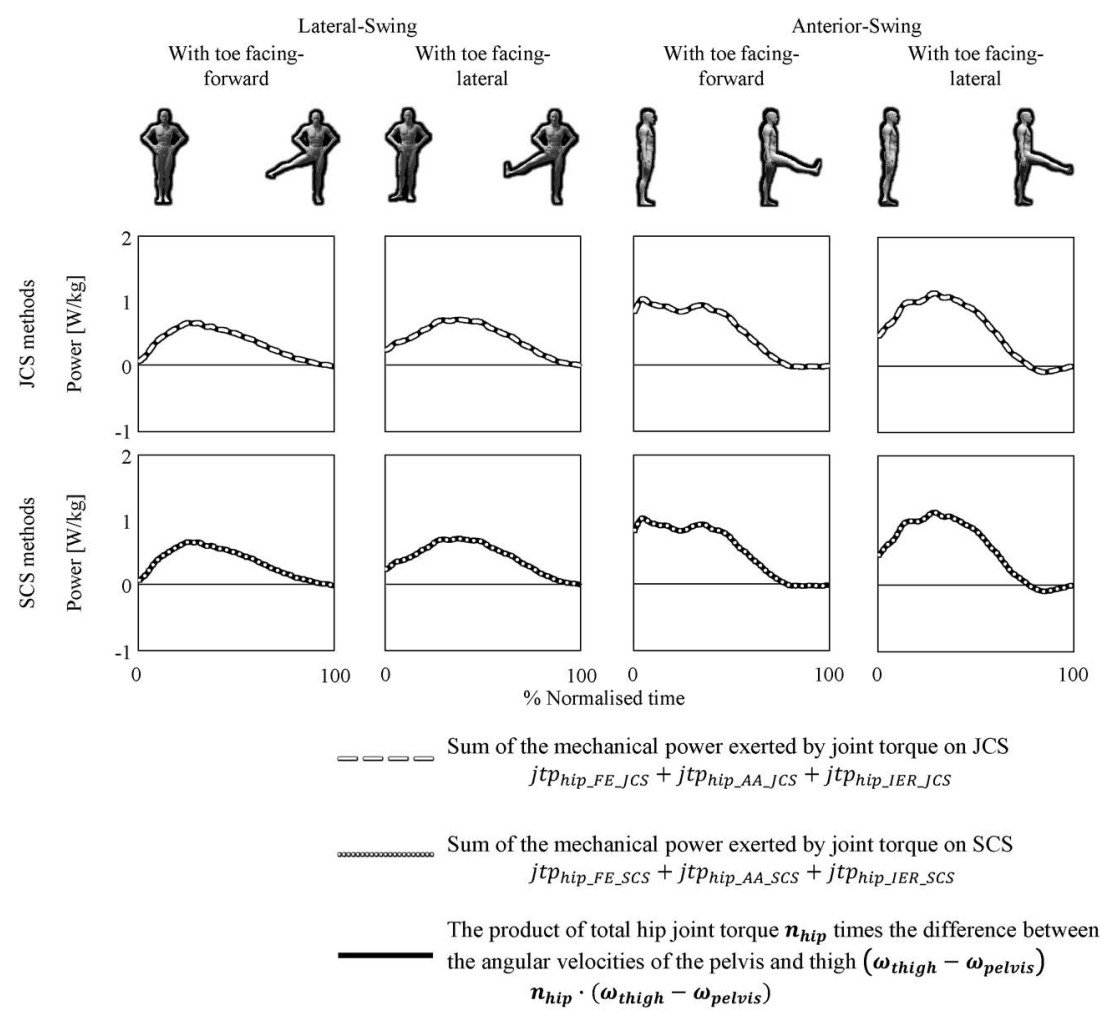

Fig. 4 Ensemble averages of the sum of the mechanical power exerted by joint torque on JCS and the product of total hip joint torque and the difference between the angular velocities of pelvis and thigh.

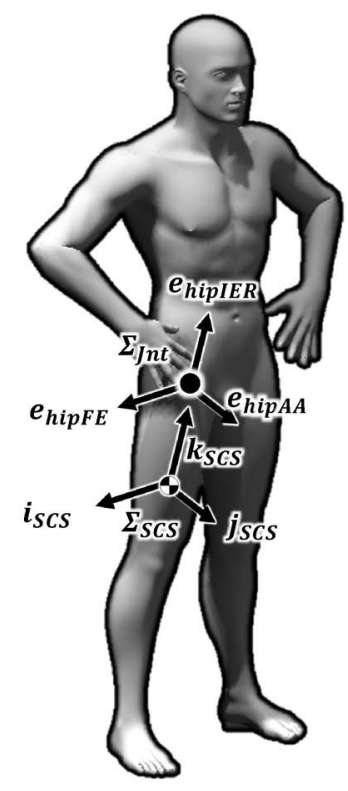

Neutral position

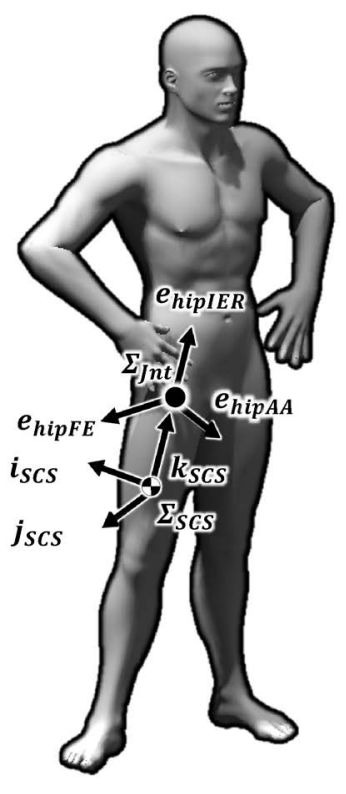

Hip external rotation position
Fig. 5 Explanation of the generation of axes difference between JCS and SCS by axial rotation.

a joint torque is thought to rotate a joint and JCS is used to describe the joint rotation, the JCS method would be recommended to investigate the power generation/absorption of each joint torque, particularly when long axial rotation occurs.

The specific muscles required to generate each anatomical torque would depend entirely upon the joint position. For example, the muscle activities of not only the iliacus but also the hip adductor muscles were observed during the follow-through phase in the side-foot soccer kick (Brophy et al., 2007). The kinematic findings of our study indicated that a similar movement (anterior swing with the toe facing laterally) mainly consisted of hip flexion (Fig. 2). The findings by Brophy et al. (2007), and those produced during the present study, can be interpreted in the sense that the hip adductor muscles generate a torque which flexes the hip joint in the hip external rotation position, although the hip adductor muscles adduct the hip joint in the anatomical position. Considering that joint torque is the net mechanical effect rotating a joint (Kristianslund et al., 2014; Schache and Baker, 2007), it would be straightforward to define the torque flexing hip joint as "flexion torque," as defined by the JCS methodology.

The hip long axial rotation of $17.8^{\circ}-51.3^{\circ}$ is observed in many dynamic sports movements such as 
baseball pitching (Kageyama et al., 2014), golf swing (Kim et al., 2014) and side-foot soccer kick (Kawamoto et al., 2007). In addition, although sprinting is considered to be a sagittal movement, it is observed that the hip internal-external rotation angle during the first and second steps in sprint running varied by $17^{\circ}$ and $20^{\circ}$, respectively (Debaere et al., 2013). Therefore, it is suggested that the JCS method is useful for understanding the biomechanical basis of various dynamic human movements.

Although the current study investigated the hip joint, shoulder joint also has three degrees of rotational freedom. For example, the throwing motion has an axial rotation of $80^{\circ}$ (Feltner and Dapena, 1986). Therefore, it was suggested that the effect of the choice of the coordinate system is larger in the shoulder than in the hip joint and that the application of the JCS method to the upper-limb movement is effective in investigating the contribution of each joint torque to power generation/absorption.

The current study had a limitation which needs to be discussed and taken into consideration. In lateral swing, the hip joint is not only abducted but is also flexed (Fig. 2). This would have occurred because the pelvis rotated toward the side of the swinging leg when the leg was swung laterally. This would have had an effect on our results; however, during lateral swing, the displacement of the hip flexion angle was much smaller than the hip abduction angle under both hip conditions, suggesting that the lateral swing would mainly consist of hip abduction movement. Therefore, the effect of this limitation would not be critical to our conclusion.

In summary, this study aims at determining the advantages of JCS in calculating the power of each anatomical torque through a comparison with SCS. The current study confirmed that using JCS for calculating the power exertion of each anatomical torque can avoid the contradiction between joint motion and power exertion, regardless of leg position, and suggested that the JCS method should be used to investigate the power generation/absorption of each joint torque when long axial rotation occurs.

\section{Conflicts of interest statement}

There are no financial or personal relationships with other people or organizations that has inappropriately influence our work.

\section{Acknowledgements}

This work was supported by a Grant-in-Aid for JSPS Research Fellow grant number 16J08165.

\section{References}

Bezodis, I. N., Kerwin, D. G., and Salo, A. I. T. (2008). Lower-limb mechanics during the support phase of maximum-velocity sprint running. Med. Sci. Sports Exerc. 40, 707-715.

Brophy, R. H., Backus, S. I., Pansy, B. S., Lyman, S., and Williams, R. J. (2007). Lower Extremity Muscle Activation and Alignment During the Soccer Instep and Side-foot Kicks. J. Orthop. Sport. Phys. Ther. 37, 260-268.

Debaere, S., Delecluse, C., Aerenhouts, D., Hagman, F., and Jonkers, I. (2013). From block clearance to sprint running: Characteristics underlying an effective transition. J. Sports Sci. 31, 137-149.

Desroches, G., Cheze, L., and Dumas, R. (2010). Expression of joint moment in the joint coordinate system. J. Biomech. Eng. 132, 114503.

Dumas, R., Cheze, L., and Verriest, J. P. (2007). Adjustments to McConville et al. and Young et al. body segment inertial parameters. J. Biomech. 40, 543-553.

Feltner, M. and Dapena, J. (1986). Dynamics of the shoulder and elbow joints of the throwing arm during a baseball pitch. Int. J. Sport. Biomech. 2, 235-259.

Ferber, R., Davis, I. M., and Williams, D. S. (2003). Gender differences in lower extremity mechanics during running. Clin. Biomech. 18, 350-357.

Fukashiro, S. and Komi, P. V. (1987). Joint moment and mechanical power flow of the lower limb during vertical jump. Int J Sport. Med 8 Suppl 1, 15-21.

Gordon, D., Robertson, E., and Winter, D. A. (1980). Mechanical energy generation, absorption and transfer amongst segments during walking. J. Biomech. 13, 845-854.

Grood, E. S. and Suntay, W. J. (1983). A joint coordinate system for the clinical description of three-dimensional motions: application to the knee. J. Biomech. Eng. 105, 136-144.

Harrington, M. E., Zavatsky, A. B., Lawson, S. E. M., Yuan, Z., and Theologis, T. N. (2007). Prediction of the hip joint centre in adults, children, and patients with cerebral palsy based on magnetic resonance imaging. J. Biomech. 40, 595-602.

Inaba, Y., Yoshioka, S., Iida, Y., Hay, D. C., and Fukashiro, S. (2013). A Biomechanical study of side steps at different distances. J. Appl. Biomech. 29, 336-345.

Kageyama, M., Sugiyama, T., Takai, Y., Kanehisa, H., and Maeda, A. (2014). Kinematic and kinetic profiles of trunk and lower limbs during baseball pitching in collegiate pitchers. J. Sport. Sci. Med. 13, 742-750.

Kawamoto, R., Miyagi, O., Ohashi, J., and Fukashiro, S. (2007). Kinetic comparison of a side-foot soccer kick between experienced and inexperienced players. Sports Biomech. 6, 187-198.

Kim, S. -B., You, J. H., Kwon, O. -Y., and Yi, C. -H. (2014). Lumbopelvic Kinematic Characteristics of Golfers With Limited Hip Rotation. Am. J. Sports Med. 43, 113-120.

Kristianslund, E., Krosshaug, T., Mok, K. M., McLean, S., and van den Bogert, A. J. (2014). Expressing the joint moments of drop jumps and sidestep cutting in different reference frames-does it matter? J. Biomech. 47, 193-199.

Schache, A. G. and Baker, R. (2007). On the expression of 
joint moments during gait. Gait Posture 25, 440-452.

Schache, A. G., Fregly, B. J., Crossley, K. M., Hinman, R. S., and Pandy, M. G. (2008). The effect of gait modification on the external knee adduction moment is reference frame dependent. Clin. Biomech. 23, 601-608.

Wells, R. P. and Winter, D. A. (1980). Assessment of Signal and Noise in the Kinematics of normal, Pathological and Sporting Gaits. Proc. 1st Conf. Cdn. Soc. Biomech., Locomot. 92-94.

Winter, D. A. (2009). Biomechanics and Motor Control of $\mathrm{Hu}-$ man Movement, 4th ed, Hoboken, N. J: John Wiley \& Sons. John Wiley \& Sons.

Wu, G., Siegler, S., Allard, P., Kirtley, C., Leardini, A., Rosenbaum, D., Whittle, M., D'Lima, D. D., Cristofolini, L., Witte, H., Schmid, O., and Stokes, I. (2002). ISB recommendation on definitions of joint coordinate system of various joints for the reporting of human joint motion-part I: ankle, hip, and spine. J. Biomech. 35, 543-548.

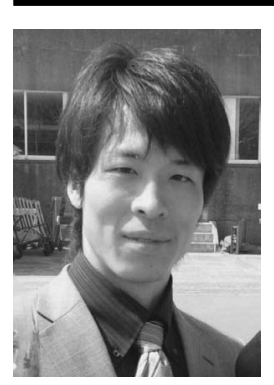

Name:

Natsuki Sado

\section{Affiliation:}

Department of Life Sciences, The University of Tokyo, Tokyo, Japan.

Research Fellow of the Japan Society for the Promotion of Science, Tokyo, Japan

\section{Address:}

3-8-1, Komaba, Meguro-ku, Tokyo, Japan; Postal code: 153-8902

\section{Brief Biographical History:}

2014 Bachelor's degree, University of Tsukuba

2016 Master's degree, The University of Tokyo.

2016-present Department of Life Sciences, The University of Tokyo, Tokyo, Japan.

2016-present Research Fellow of the Japan Society for the Promotion of Science, Tokyo, Japan

\section{Main Works:}

-Sado, N., Yoshioka, S., and Fukashiro, S., 2017. The threedimensional kinetic behaviour of the pelvic rotation in maximal sprint running. Sport. Biomech. 16, 258-271.

-Sado, N., Yoshioka, S., and Fukashiro, S., 2016. Mechanism of the maintenance of sagittal trunk posture in maximal sprint running. Jpn J Biomech Sports Exerc 20, 56-64.

Membership in Learned Societies:

-Japan Society of Physical Education, Health and Sport Sciences

- Japanese Society of Biomechanics

-Japan Society of Training Science for Exercise and Sport 